\title{
Seasonal Incidence of Major Pests of Pongamia (Milletia pinnata L.) in Nursery Conditions
}

\author{
D. Devika Rani*, D. Jemla Naik and K.S. Jagadish
}

Department of Agricultural Entomology, UAS, GKVK, Bengaluru - 560065, India

*Corresponding author

\section{Keywords \\ Pongamia, Aceria pongamiae, Leaf galls, Flower galls, Weather parameters \\ Article Info \\ Accepted: \\ 12 July 2018 \\ Available Online: \\ 10 August 2018}

\section{A B S T R A C T}

The incidence of major pests of pongamia in relation to weather parameters was observed during 2016-17 at Zonal Agricultural Research Station, UAS, GKVK, Bengaluru. The important pests recorded on pongamia were blotch miner (Acrocercops anthrauris Fabricius), spiralling whitefly Aleurodiccus disperses (Russel), and the eriophyid mite, Aceria pongamiae Channabasavanna causing leaf galls. Correlation between A. pongamiae with weather variables of the corresponding fortnight revealed that it had highly significant and positive correlation with minimum temperature i.e. $\left(\mathrm{r}=0.845^{* *}, \mathrm{p}<0.01\right)$, maximum temperature $(r=0.471 *, p<0.05)$ and total rainfall $(r=0.464 *)$ respectively. Leaf gall number was significantly and positively correlated with minimum temperature $(\mathrm{r}=0.605$, $\mathrm{p}<0.01)$. Regarding correlation with preceding fortnight weather data, A. pongamiae incidence was significantly and positively correlated with maximum temperature $(\mathrm{r}=0$. $571, \mathrm{p}<0.01)$ and minimum temperature $(\mathrm{r}=0.868, \mathrm{p}<0.01)$, leaf gall number due to $A$. pongamiae was significantly and positively correlated with minimum temperature $(\mathrm{r}=$ $\left.0.586^{* *}, \mathrm{p}<0.01\right)$, total rainfall $(\mathrm{r}=0.478, \mathrm{p}<0.05)$ and wind speed $\left(\mathrm{r}=0.508^{*}, \mathrm{p}<0.05\right)$. Blotch miner incidence was significantly and positively correlated with maximum temperature $(\mathrm{r}=0.413, \mathrm{p}<0.01)$, minimum temperature $(\mathrm{r}=0.684, \mathrm{p}<0.01)$, wind speed $(\mathrm{r}$ $\left.=0.361^{* *}, \mathrm{p}<0.01\right)$, afternoon RH $(\mathrm{r}=0.290, \mathrm{p}<0.05)$ and total rainfall $(\mathrm{r}=0.292, \mathrm{p}<0.05)$ of the preceding week. Spiralling whitefly incidence was significantly positively correlated with maximum temperature $(\mathrm{r}=0.072, \mathrm{p}<0.01)$, minimum temperature i.e. $(\mathrm{r}=0.517$, $\mathrm{p}<0.01)$ and afternoon RH $(\mathrm{r}=0.520, \mathrm{p}<0.01)$. Regarding correlation with the corresponding week weather parameters, blotch miner showed significant and positive correlation with minimum temperature $(\mathrm{r}=0.775, \mathrm{p}<0.01)$, maximum temperature $(\mathrm{r}=$ $0.289, \mathrm{p}<0.05)$, afternoon relative humidity $(\mathrm{r}=0.336, \mathrm{p}<0.05)$ and wind speed $(\mathrm{r}=0.359$, $\mathrm{p}<0.01)$. Spiralling whitefly, incidence showed significant positive correlation with maximum temperature $(\mathrm{r}=0.069, \mathrm{p}<0.01)$, minimum temperature i.e. $\left(\mathrm{r}=0.523^{* *}, \mathrm{p}<0.01\right)$ morning $\mathrm{RH}(\mathrm{r}=0.277, \mathrm{p}<0.05)$ afternoon $\mathrm{RH}\left(\mathrm{r}=0.536^{* *}, \mathrm{p}<0.01\right)$ and rainfall $(\mathrm{r}=$ $0.353, \mathrm{p}<0.05)$

\section{Introduction}

Pongamia (Milletia pinnata L.), belongs to family Fabaceae, is a medium-sized evergreen or briefly deciduous, glabrous shrub or tree normally growing to a height of $15-25 \mathrm{~m}$ high, with straight or a crooked trunk of 50-80 $\mathrm{cm}$ diameter and broad crown of spreading or 
drooping branches ${ }^{[7]}$ It is a medium sized glabrous tree, mainly distributed in tidal forests of India. Recent recognition of the importance of the seeds of this plant as a raw material for biofuel production extends its economic utility for industrial applications also.

The non-edible oil contains several unsaponifiable and toxic components, which make them unsuitable for human consumption. Karanja (Pongamia pinnata) is an underutilized plant which is grown in many parts of India. Sometimes the oil is contaminated with high free fatty acids (FFA) depending upon the moisture content in the seed during collection as well as oil expression ${ }^{[3]}$. The eriophyid mite $A$. pongamiae Channabasavanna and flower galls caused due to $A$. pongamiae Mani are the major pests. A. pongamiae feeds on the leaves which lead to the formation of elongate finger-like pouches of varying dimensions on both surfaces of the leaves. Individual galls fuse to form complex, irregularly shaped, massive structures, covering the entire laminar area including the midrib, vein and veinlets. This results in severe distortion of the leaves and drastically affects the photosynthetic activity of the plant, leading to reduction of biomass and adversely affects the growth of $P$. pinnata. The pest showed a higher percentage of damage and number of galls during May, leading to a significant reduction in leaf area [4]. Growth attributes viz., length, collar girth and biomass in treated (non-infested) seedlings are significantly higher than the untreated (infested) seedlings of $P$. pinnata, $(\mathrm{p}<0.001)^{[5]}$. However, flower galls caused by $A$. pongamiae, are also important due to its negative impact on seed set ${ }^{[8]}$. The present investigation focuses on the population dynamics of major pests of Pongamia in relation to the climatic factors, the findings of which are discussed in the light of earlier reports on the similar type of investigations.
Some important natural enemies observed on P. Pinnata was Green lacewing, Chrysoperla sp. Mantids, Spiders, Predatory red stink bug, Euthyrhynchus floridanus Linnaeus, Lady bird beetle ${ }^{[6]}$.

\section{Material and Methods}

\section{Incidence of eriophyid mite, Aceria} pongamiae and Leaf gall

For the purpose of recording the incidence of eriophyid mite, A. pongamiae and leaf gall it causes on Pongamia, fifteen galled leaf samples were collected randomly from Pongamia and observations were carried out for one year, at fortnightly intervals. The leaf samples were put in polythene bags and tied loosely with rubber bands for subsequent transportation to the laboratory for further observation under the microscope. Data was recorded on the number of galls developed on both abaxial and adaxial surface of the leaves. Data on temperature, relative humidity, and rainfall of the study site were obtained from AICRP (Agrometeorology), UAS, GKVK, Bengaluru. The incidence of $A$. pongamiae and the number of leaf galls was subjected to correlation and multiple linear regression analysis with the weather parameters of the corresponding fortnight and also with that of the preceding fortnight.

\section{Incidence of blotch miner and spiralling whitefly caused by Acrocercops anthrauris Fabricius}

The data pertaining to the incidence of insect pests of blotch miner, spiralling whitefly and flower galls on Pongamia were recorded at weekly interval for a period of one year. Fifteen trees were labelled and observed for the incidence of these three pests at weekly intervals. The observation on the pest population was recorded from five upper, five middle and five lower leaves, in each of the fifteen plants in each replication. The same 
set of labelled plants was used for continuously recording observations during the study period. Incidence of blotch miner, spiralling whitefly and flower galls was subjected to correlation and multiple linear regression analysis with weather parameters of both preceeding and corresponding week.

Weather parameters were mentioned in regression tables as

$\mathbf{R}_{\mathbf{1}}=$ Maximum temperature $\left({ }^{0} \mathrm{C}\right)$

$\mathbf{R}_{\mathbf{2}}=$ Minimum temperature $\left({ }^{0} \mathrm{C}\right)$

$\mathbf{R}_{\mathbf{3}}=$ Relative Humidity morning $(\%)$

$\mathbf{R}_{\mathbf{4}}=$ Relative Humidity afternoon $(\%)$

$\mathbf{R}_{\mathbf{5}}=$ Wind speed $(\mathrm{Km} /$ day $)$

$\mathbf{R}_{\mathbf{6}}=$ Bright sunshine (Hrs)

$\mathbf{R}_{7}=$ Total Rainfall (mm)

\section{Results and Discussion}

Incidence of Aceria pongamiae and leaf galls in relation to weather parameters in nursery

The results pertaining to the incidence of major pests of pongamia (Milletia pinnata L.) are discussed below. Maximum mite (Aceria pongamiae) population was observed in II fortnight of May (198.92), followed by I fortnight of May (179.30). No mite population was observed in the entire month of February and I fortnight of January (Table 1).

Maximum number of leaf galls due to Aceria pongamiae infestation was observed during II fortnight of June (66.76), followed by I fortnight of June (59.71). No leaf gall infestation was observed during II fortnight of January and I fortnight of February (Table 1).

From this results we can conclude that the number of gall to be highest during May. Population studies carried out on $A$. pongamiae enabled to record seasonal fluctuations in the mite density within the leaf galls. The initiation of infestation was recorded in February, reaching to peak population in May. The decline in mite population since June would be reflecting the negative impact of rain fall received by the site. There is no mite population and galls recorded on Pongamia in January and February because of shedding of their leaves. Even though the mites enjoy a highly secluded habitat available within the leaf galls, rain fall exerts an adverse effect by penetrating through the holes in to the interior of the gall cavity ${ }^{[1] \text {. }}$

Our results have been found to be in conformity with the findings of (Aratchige et al., 2012) who reported a significant negative impact of rainfall on the population density of Neoseiulus baraki., low number of mites recorded inside the dried galls during October and November could be possibly explained on the grounds of their escape in search of suitable sites like the under surfaces of the bark or to hibernate/or tide over the unfavourable conditions through the phenomenon of deuterogyny. The plants shed the leaves in January. The trend observed on the population fluctuation of $A$. pongamiae has been found to be in accordance with the findings of who reported maximum population density of $A$. litchi during AprilMay and minimum during NovemberDecember. Another study also recorded similar trends in population of Aculus euphorbiae feeding on euphorbia spp. recording a lower population in winter ${ }^{[4] \text {. }}$

\section{Correlation between incidence of Aceria pongamiae and leaf galls with weather parameters of preceding fortnight in nursery}

The correlation between eriophyid mite population with weather parameters of preceding fortnight was found to be highly 
significant and positively correlated with maximum temperature $(r=0.571, \mathrm{p}<0.01)$ and minimum temperature $(\mathrm{r}=0.868$, $\mathrm{p}<0.01$ ), while it was non - significant and positive with wind speed $(r=0.166)$ total rainfall $(\mathrm{r}=0.531)$ and afternoon $\mathrm{RH}(\mathrm{r}=$ 0.173).

However, mite incidence was negatively correlated with morning RH $(r=-0.071)$, and sunshine hours $(r=-0.313)$.

The correlation between number of leaf galls caused by mite with weather parameters of preceding fortnight was found to be significantly positively correlated with minimum temperature $\left(\mathrm{r}=0.586^{* *}, \mathrm{p}<0.01\right)$, total rainfall $(\mathrm{r}=0.478, \mathrm{p}<0.05)$ and wind speed $\left(\mathrm{r}=0.508^{*}, \mathrm{p}<0.05\right)$. However, gall incidence was found to have non - significant positive correlation with maximum temperature $(\mathrm{r}=0.352)$, morning $\mathrm{RH}(\mathrm{r}=$ $0.027)$ and afternoon $\mathrm{RH}(\mathrm{r}=0.159)$. It was non - significantly negatively correlated with sunshine hrs. $(r=-0.276)$.

Correlation between incidence of Aceria pongamiae and leaf galls with weather parameters of corresponding fortnight in Nursery

The correlation analysis between eriophyid mite population with weather parameters of corresponding fortnight was found to be significantly positively correlated with minimum temperature i.e. $\left(\mathrm{r}=0.845^{* *}\right.$, $\mathrm{p}<0.01)$, maximum temperature $\left(\mathrm{r}=0.471^{*}\right.$, $\mathrm{p}<0.05)$ and total rainfall $(\mathrm{r}=0.464 *)$ and mite population showed non - significant positive correlation with afternoon $\mathrm{RH}(\mathrm{r}=0$. 212), wind speed $(r=0.200)$ and Negative correlation was recorded between morning RH ( $r=-0.060)$, sunshine hrs $(r=-0.301)$ with mite incidence, but this relationship was found to be non - significant.
The correlation between incidence of leaf galls with weather parameters of corresponding fortnight was found to be significant and positively correlated with minimum temperature $(\mathrm{r}=0.605, \mathrm{p}<0.01)$, while it showed non - significant positive correlation with maximum temperature $(\mathrm{r}=$ 0.199), morning RH ( $\mathrm{r}=0.136)$, afternoon RH $(r=0.225)$ wind speed $(r=0.043)$ and total rainfall $(\mathrm{r}=0.155)$ (Table 2 and 3$)$.

However, leaf gall number was negatively correlated with sunshine hours $(r=-0.446)$ and this relationship was non - significant.

Multiple linear regression between incidence of Aceria pongamiae and leaf galls with weather parameters of preceding fortnight in Nursery

Multiple linear regression analysis revealed that the weather parameters of preceding fortnight influenced $A$. pongamiae and leaf gall formation to an extent of 87.9 and 53.4 per cent, respectively. ${ }^{[1,4]}$ also reported a similar impact of weather parameters on leaf galls and eriophyid mites (Table 4).

Multiple linear regression between incidence of Aceria pongamiae and leaf galls with weather parameters of corresponding fortnight in Nursery

Multiple linear regression analysis revealed that the weather parameters of corresponding fortnight influenced $A$. pongamiae and leaf gall formation to an extent of 77.10 and 45.10 per cent, respectively (Table 5).

[1] and [4] also reported about weather parameters influencing leaf galls and eriophyid mites to an extent of 78.3 and 50.28 per cent, respectively, which was similar to the present findings. 
Table.1 Incidence of Aceria pongamiae and leaf galls caused by A. pongamiae in relation to weather parameters of the preceding fortnight (2016-17) (Nursery)

\begin{tabular}{|c|c|c|c|c|c|c|c|c|c|c|c|}
\hline \multirow{2}{*}{$\begin{array}{l}\text { Fort night of } \\
\text { eachMonth }\end{array}$} & \multirow{2}{*}{$\begin{array}{l}\text { Std. } \\
\text { Met. } \\
\text { week }\end{array}$} & \multirow{2}{*}{$\begin{array}{c}\text { Date of } \\
\text { Observation }\end{array}$} & \multirow{2}{*}{$\begin{array}{l}\text { No of } \\
\text { A.Pongamiael } \\
\text { gall }\end{array}$} & \multirow{2}{*}{$\begin{array}{c}\text { Leaf } \\
\text { galls(No } \\
\text { /leaf) }\end{array}$} & \multicolumn{2}{|c|}{ Temp $\left({ }^{0} \mathrm{C}\right)$} & \multicolumn{2}{|c|}{ RH (\%) } & \multirow{2}{*}{$\begin{array}{c}\text { Wind } \\
\text { speed } \\
(\mathrm{Km} / \mathrm{day})\end{array}$} & \multirow{2}{*}{$\begin{array}{l}\text { Sunshine } \\
\text { (Hrs) }\end{array}$} & \multirow{2}{*}{$\begin{array}{c}\text { Total } \\
\text { Rainfall } \\
(\mathbf{m m})\end{array}$} \\
\hline & & & & & Max. & Min. & Morning & $\mathrm{A} / \mathrm{N}$ & & & \\
\hline I FN. Oct & 40 & $3-10-2016$ & 146.73 & 7.26 & 28.89 & 19.05 & 93.15 & 50.86 & 6.20 & 5.40 & 16.40 \\
\hline II FN. Oct & 42 & $17-10-2016$ & 119.24 & 11.41 & 29.42 & 18.76 & 84.93 & 48.07 & 4.45 & 7.05 & 30.20 \\
\hline I FN. Nov & 45 & 7-11-2016 & 109.52 & 13.35 & 29.68 & 17.76 & 80.86 & 46.50 & 3.70 & 8.80 & 0.00 \\
\hline II FN. Nov & 47 & $21-11-2016$ & 78.65 & 12.08 & 29.33 & 17.20 & 81.43 & 45.36 & 6.20 & 8.40 & 0.00 \\
\hline IFN. Dec & 49 & $5-12-2016$ & 67.56 & 6.64 & 29.69 & 21.80 & 82.29 & 40.22 & 5.35 & 8.70 & 1.70 \\
\hline II FN. Dec & 51 & $19-12-2016$ & 10.32 & 4.32 & 26.09 & 15.36 & 86.22 & 49.57 & 7.45 & 4.70 & 58.20 \\
\hline I FN. Jan & 2 & $9-01-2017$ & 3.65 & 2.32 & 28.03 & 13.76 & 89.07 & 48.57 & 5.30 & 9.15 & 0.000 \\
\hline II FN. Jan & 4 & $23-01-2017$ & 0.00 & 0.00 & 27.30 & 13.64 & 88.5 & 41.43 & 5.15 & 8.80 & 0.00 \\
\hline I FN. Feb & 6 & $6-02-2017$ & 0.00 & 0.00 & 27.03 & 14.76 & 89.36 & 39.00 & 9.10 & 7.60 & 0.00 \\
\hline II FN. Feb & 8 & $20-02-2017$ & 0.00 & 3.66 & 28.59 & 14.66 & 84.22 & 36.29 & 5.65 & 10.40 & 0.00 \\
\hline I FN. Mar & 11 & $13-03-2017$ & 34.50 & 7.07 & 30.69 & 15.20 & 84.29 & 34.79 & 8.05 & 9.95 & 1.40 \\
\hline II FN. Mar & 13 & $27-03-2017$ & 25.40 & 9.10 & 32.13 & 17.55 & 82.64 & 35.22 & 6.05 & 9.15 & 0.00 \\
\hline I FN. Apr & 15 & 9-04-2017 & 149.86 & 10.87 & 32.83 & 20.22 & 81.00 & 41.00 & 6.70 & 8.95 & 1.60 \\
\hline II FN. Apr & 17 & 23-04-2017 & 161.29 & 13.98 & 34.80 & 21.00 & 82.00 & 36.50 & 6.95 & 8.95 & 0.00 \\
\hline I FN. May & 19 & $7-05-2017$ & 179.30 & 22.56 & 35.15 & 22.15 & 83.00 & 36.00 & 7.55 & 8.10 & 41.00 \\
\hline II FN. May & 21 & $21-05-2017$ & 198.92 & 36.52 & 34.05 & 21.65 & 83.00 & 33.50 & 6.50 & 8.50 & 179.20 \\
\hline I FN. Jun & 23 & 4-06-2017 & 172.83 & 59.71 & 33.05 & 21.05 & 83.00 & 41.50 & 7.35 & 7.45 & 29.90 \\
\hline II FN. Jun & 25 & $18-06-2017$ & 169.80 & 66.76 & 31.10 & 19.85 & 87.50 & 44.50 & 9.40 & 7.35 & 40.60 \\
\hline I FN. Jul & 27 & $2-07-2017$ & 101.43 & 43.12 & 28.80 & 20.40 & 89.00 & 55.00 & 10.55 & 4.55 & 13.40 \\
\hline II FN. Jul & 29 & $16-07-2017$ & 94.23 & 34.75 & 28.20 & 19.75 & 88.50 & 55.00 & 11.15 & 4.95 & 16.00 \\
\hline I FN. Aug & 31 & 01-08-2017 & 112.21 & 32.85 & 28.50 & 19.75 & 91.50 & 59.00 & 9.70 & 3.95 & 77.40 \\
\hline II FN. Aug & 34 & $16-08-2017$ & 159.45 & 23.67 & 28.70 & 19.65 & 86.50 & 54.50 & 11.60 & 6.05 & 97.00 \\
\hline I FN. Sep & 36 & $05-09-2017$ & 155.86 & 16.89 & 28.05 & 19.90 & 91.00 & 56.00 & 5.85 & 3.35 & 130.00 \\
\hline II FN. Sep & 38 & $19-09-2017$ & 149.53 & 13.08 & 27.50 & 19.70 & 91.00 & 61.50 & 7.25 & 3.35 & 48.60 \\
\hline
\end{tabular}


Table. 2 Correlation between incidence of Aceria pongamiae and leaf galls with weather parameters of the preceding fortnight (Nursery)

\begin{tabular}{|c|c|c|c|c|c|c|c|}
\hline \multirow{2}{*}{ Mite / leaf gall } & \multicolumn{2}{|c|}{ Temp $\left({ }^{0} \mathrm{C}\right)$} & \multicolumn{2}{|c|}{ RH (\%) } & \multirow{2}{*}{$\begin{array}{c}\text { Wind } \\
\text { speed } \\
(\mathrm{Km} / \text { day })\end{array}$} & \multirow{2}{*}{$\begin{array}{l}\text { Bright } \\
\text { Sunshine } \\
\text { (Hrs) }\end{array}$} & \multirow{2}{*}{$\begin{array}{l}\text { Total } \\
\text { Rainfall } \\
(\mathrm{mm})\end{array}$} \\
\hline & Max. & Min. & Morning & $\mathbf{A} / \mathbf{N}$ & & & \\
\hline $\begin{array}{c}\text { Aceria } \\
\text { pongamiae }\end{array}$ & $0.571^{* *}$ & $0.868 * *$ & & 0.173 & 0.184 & -0.313 & 0.531 \\
\hline Leaf galls & 0.352 & $0.586^{* *}$ & 0.027 & 0.159 & $0.508^{*}$ & -0.276 & 0.347 \\
\hline
\end{tabular}

Table.3 Correlation between incidence of Aceria pongamiae and leaf galls with weather parameters of the corresponding fortnight (Nursery)

\begin{tabular}{|c|c|c|c|c|c|c|c|}
\hline \multirow[b]{2}{*}{ Mite / leaf gall } & \multicolumn{2}{|c|}{ Temp $\left({ }^{0} \mathrm{C}\right)$} & \multicolumn{2}{|c|}{$\mathrm{RH}(\%)$} & \multirow{2}{*}{$\begin{array}{c}\text { Wind speed } \\
\text { (Km/day) }\end{array}$} & \multirow{2}{*}{$\begin{array}{c}\text { Bright } \\
\text { Sunshine } \\
\text { (Hrs) }\end{array}$} & \multirow{2}{*}{$\begin{array}{l}\text { Total } \\
\text { Rainfall } \\
(\mathrm{mm})\end{array}$} \\
\hline & Max. & Min. & Morning & $\mathbf{A} / \mathbf{N}$ & & & \\
\hline Aceria pongamiae & $0.471^{*}$ & $0.845^{* *}$ & -0.060 & 0.212 & 0.200 & -0.301 & $0.464^{*}$ \\
\hline Leaf galls & 0.199 & $0.605 * *$ & 0.136 & 0.225 & 0.043 & -0.446 & 0.155 \\
\hline
\end{tabular}

Table.4 Multiple linear regression between incidence of Aceria pongamiae and leaf galls with weather parameters of the preceding fortnight (Nursery)

\begin{tabular}{|l|l|l|}
\hline Mite / leafgall & \multicolumn{1}{|c|}{ Regression equation } & $\mathrm{R}^{2}$ \\
\hline Aceria pongamiae & $\begin{array}{l}\mathrm{Y}=-1003.141+16.907 \mathrm{R}_{1}+10.452 \mathrm{R}_{2}+2.052 \mathrm{R}_{3}+87.90 \\
4.308 \mathrm{R}_{4}-1.933 \mathrm{R}_{5}+4.780 \mathrm{R}_{6}+0.346 \mathrm{R}_{7}\end{array}$ \\
\hline Leaf gall & $\begin{array}{l}\mathrm{Y}=-183.755+2.846 \mathrm{R}_{1}+1.521 \mathrm{R}_{2}+0.220 \mathrm{R}_{3}+53.40 \\
0.707 \mathrm{R}_{4}+3.586 \mathrm{R}_{5}+1.478 \mathrm{R}_{6}+0.053 \mathrm{R}_{7}\end{array}$ & \\
\hline
\end{tabular}

Table.5 Multiple linear regression between incidence of Aceria pongamiae and leaf galls with weather parameters of the corresponding fortnight (Nursery)

\begin{tabular}{|l|l|r|}
\hline \multicolumn{1}{|c|}{ Mite / leaf gall } & \multicolumn{1}{|c|}{ Regression equation } & $R^{2}$ \\
\hline Aceria pongamiae & $Y=-165.878-2.760 R_{1}+22.354 R_{2}-2.510 R_{3}+$ & 77.10 \\
& $2.144 R_{4}-1.634 R_{5}+8.632 R_{6}+0.208 R_{7}$ & \\
\hline Leaf gall & $Y=-19.401-0.826 R_{1}+4.533 R_{2}+0.031 R_{3}-0.104$ & 45.10 \\
& $R_{4}+0.178 R_{5}-2.168 R_{6}-0.068 R_{7}$ & \\
\hline
\end{tabular}


Table.6 Incidence of blotch miner and spiralling whitefly caused by Acrocercops anthrauris Fabricius in relation to weather parameters of the preceding week during 2016-17 (Nursery)

\begin{tabular}{|c|c|c|c|c|c|c|c|c|c|c|}
\hline \multirow{2}{*}{$\begin{array}{c}\text { Date of } \\
\text { observation }\end{array}$} & \multirow{2}{*}{$\begin{array}{l}\text { Std. Met. } \\
\text { week }\end{array}$} & \multirow{2}{*}{$\begin{array}{c}\text { No. of } \\
\text { blotches/ } \\
\text { leaf }\end{array}$} & \multirow{2}{*}{$\begin{array}{l}\text { No. of egg } \\
\text { spirals of } \\
\text { SWF/leaf }\end{array}$} & \multicolumn{2}{|c|}{ Temp $\left({ }^{0} \mathrm{C}\right)$} & \multicolumn{2}{|c|}{$\mathrm{RH}(\%)$} & \multirow{2}{*}{$\begin{array}{l}\text { Wind speed } \\
(\mathrm{Km} / \mathrm{day})\end{array}$} & \multirow{2}{*}{$\begin{array}{c}\text { Bright } \\
\text { Sunshine } \\
\text { (Hrs) }\end{array}$} & \multirow{2}{*}{$\begin{array}{l}\text { Total } \\
\text { Rainfall } \\
(\mathrm{mm})\end{array}$} \\
\hline & & & & Max. & Min. & Morning & $\mathbf{A} / \mathbf{N}$ & & & \\
\hline $3-10-2016$ & 40 & 17.72 & 3.13 & 27.86 & 19.20 & 94.29 & 54.43 & 6.80 & 2.50 & 16.40 \\
\hline $10-10-2016$ & 41 & 17.83 & 3.63 & 29.91 & 18.91 & 92.00 & 47.29 & 5.60 & 8.30 & 0.00 \\
\hline $17-10-2016$ & 42 & 18.03 & 3.01 & 29.09 & 18.66 & 92.00 & 50.57 & 5.50 & 4.90 & 30.2 \\
\hline 24-10-2016 & 43 & 18.39 & 3.43 & 29.74 & 16.69 & 77.86 & 45.57 & 3.40 & 9.20 & 0.00 \\
\hline $31-10-2016$ & 44 & 17.49 & 2.63 & 29.66 & 17.40 & 76.71 & 45.86 & 3.70 & 8.80 & 0.00 \\
\hline 7-11-2016 & 45 & 16.64 & 2.61 & 29.69 & 18.11 & 85.00 & 47.14 & 3.70 & 8.80 & 0.00 \\
\hline $14-11-2016$ & 46 & 15.83 & 2.53 & 29.20 & 15.97 & 78.71 & 45.00 & 4.70 & 9.60 & 0.00 \\
\hline 21-11-2016 & 47 & 14.99 & 2.47 & 29.46 & 18.43 & 84.14 & 45.71 & 7.70 & 7.20 & 0.00 \\
\hline 28-11-2016 & 48 & 13.53 & 2.41 & 29.89 & 29.60 & 82.00 & 38.43 & 6.70 & 8.70 & 0.00 \\
\hline $5-12-2016$ & 49 & 10.24 & 2.39 & 29.49 & 14.00 & 82.57 & 42.00 & 4.00 & 8.70 & 1.70 \\
\hline 12-12-2016 & 50 & 7.93 & 2.32 & 27.37 & 15.60 & 82.57 & 43.14 & 7.70 & 4.70 & 0.00 \\
\hline $19-12-2016$ & 51 & 2.95 & 2.23 & 24.80 & 15.11 & 89.86 & 56.00 & 7.20 & 4.70 & 58.20 \\
\hline $26-12-2016$ & 52 & 1.99 & 2.17 & 28.14 & 13.91 & 87.43 & 41.14 & 5.50 & 8.60 & 0.00 \\
\hline 2-01-2017 & 1 & 1.01 & 1.91 & 27.91 & 13.6 & 90.71 & 39.29 & 5.10 & 9.70 & 0.00 \\
\hline 9-01-2017 & 2 & 0.00 & 1.63 & 27.37 & 12.17 & 85.86 & 41.00 & 5.20 & 9.40 & 0.00 \\
\hline 16-01-2017 & 3 & 0.00 & 0.00 & 27.23 & 15.11 & 91.14 & 41.86 & 5.10 & 8.20 & 0.00 \\
\hline 23-01-2017 & 4 & 0.00 & 0.00 & 26.77 & 14.09 & 93.14 & 39.43 & 8.50 & 9.40 & 0.00 \\
\hline 30-01-2017 & 5 & 0.00 & 0.00 & 27.29 & 15.43 & 85.57 & 38.57 & 9.70 & 5.80 & 0.00 \\
\hline 6-02-2017 & 6 & 0.00 & 0.00 & 27.29 & 15.43 & 85.57 & 38.57 & 4.80 & 10.50 & 0.00 \\
\hline 13-02-2017 & 7 & 0.21 & 0.00 & 29.89 & 13.89 & 82.86 & 34.00 & 6.50 & 10.30 & 0.00 \\
\hline 20-02-2017 & 8 & 3.94 & 0.00 & 29.43 & 14.63 & 83.86 & 33.86 & 10.20 & 10.00 & 0.00 \\
\hline 27-02-2017 & 9 & 7.82 & 0.00 & 31.94 & 15.77 & 84.71 & 35.71 & 5.90 & 9.90 & 1.40 \\
\hline 06-03-2017 & 10 & 13.33 & 1.05 & 31.97 & 15.03 & 78.71 & 29.14 & 5.90 & 9.90 & 0.00 \\
\hline
\end{tabular}




\begin{tabular}{|c|c|c|c|c|c|c|c|c|c|c|}
\hline \multirow{2}{*}{$\begin{array}{c}\text { Date of } \\
\text { observation }\end{array}$} & \multirow{2}{*}{$\begin{array}{c}\text { Std. Met. } \\
\text { week }\end{array}$} & \multirow{2}{*}{$\begin{array}{c}\text { No. of } \\
\text { blotches/ } \\
\text { leaf }\end{array}$} & \multirow{2}{*}{$\begin{array}{l}\text { No. of egg } \\
\text { spirals of } \\
\text { SWF/leaf }\end{array}$} & \multicolumn{2}{|c|}{ Temp ${ }^{0} \mathrm{C}$} & \multicolumn{2}{|c|}{ RH\% } & \multirow{2}{*}{$\begin{array}{c}\text { Wind speed } \\
\text { (Km/day) }\end{array}$} & \multirow{2}{*}{$\begin{array}{c}\text { Bright } \\
\text { sunshine } \\
(\mathrm{Hrs})\end{array}$} & \multirow{2}{*}{$\begin{array}{c}\text { Total } \\
\text { Rainfal } \\
(\mathrm{mm})\end{array}$} \\
\hline & & & & Max. & Min. & Morning & $\mathbf{A} / \mathbf{N}$ & & & \\
\hline 13-03-2017 & 11 & 14.56 & 1.13 & 32.29 & 20.06 & 86.57 & 41.29 & 6.20 & 8.40 & 1.40 \\
\hline 20-03-2017 & 12 & 15.34 & 1.21 & 32.26 & 19.94 & 83.57 & 41.57 & 6.50 & 8.50 & 0.00 \\
\hline 27-03-2017 & 13 & 16.86 & 2.32 & 33.40 & 20.49 & 78.43 & 40.43 & 6.90 & 9.40 & 0.00 \\
\hline 2-04-2017 & 14 & 17.97 & 2.66 & 35.00 & 20.50 & 82.00 & 35.00 & 7.70 & 9.30 & 0.00 \\
\hline 9-04-2017 & 15 & 18.13 & 2.55 & 34.60 & 21.50 & 82.00 & 38.00 & 6.20 & 8.60 & 1.60 \\
\hline 16-04-2017 & 16 & 18.97 & 2.43 & 35.70 & 22.40 & 82.00 & 36.00 & 6.70 & 8.50 & 0.00 \\
\hline 23-04-2017 & 17 & 20.14 & 2.51 & 34.60 & 21.90 & 84.00 & 36.00 & 8.40 & 7.70 & 0.00 \\
\hline 30-04-2017 & 18 & 23.24 & 2.63 & 35.40 & 22.40 & 84.00 & 36.00 & 7.00 & 8.80 & 0.00 \\
\hline 7-05-2017 & 19 & 25.36 & 2.91 & 32.70 & 20.90 & 82.00 & 31.00 & 6.00 & 8.20 & 25.80 \\
\hline 14-05-2017 & 20 & 26.53 & 3.01 & 32.60 & 21.20 & 81.00 & 48.00 & 6.40 & 6.90 & 15.20 \\
\hline 21-05-2017 & 21 & 29.88 & 3.12 & 33.50 & 20.90 & 85.00 & 35.00 & 8.30 & 8.00 & 45.60 \\
\hline 28-05-2017 & 22 & 31.33 & 4.11 & 32.20 & 19.80 & 87.00 & 42.00 & 8.00 & 6.50 & 133.6 \\
\hline 4-06-2017 & 23 & 32.09 & 4.68 & 30.00 & 19.90 & 88.00 & 47.00 & 10.80 & 8.20 & 8.30 \\
\hline 11-06-2017 & 24 & 30.21 & 4.31 & 29.50 & 20.60 & 88.00 & 53.00 & 10.20 & 6.80 & 21.60 \\
\hline 18-06-2017 & 25 & 28.24 & 4.16 & 28.10 & 20.20 & 90.00 & 57.00 & 10.90 & 2.30 & 8.00 \\
\hline $25-06-2017$ & 26 & 26.13 & 3.32 & 28.10 & 19.50 & 90.00 & 56.00 & 9.00 & 4.80 & 32.60 \\
\hline 2-07-2017 & 27 & 25.42 & 3.41 & 28.30 & 20.00 & 87.00 & 54.00 & 13.30 & 5.10 & 3.20 \\
\hline 9-07-2017 & 28 & 24.01 & 3.29 & 29.00 & 19.70 & 93.00 & 57.00 & 9.60 & 4.20 & 10.20 \\
\hline 16-07-2017 & 29 & 22.34 & 2.11 & 28.00 & 19.80 & 90.00 & 61.00 & 9.80 & 3.70 & 13.40 \\
\hline 23-07-2017 & 30 & 20.77 & 2.04 & 27.50 & 19.60 & 88.00 & 59.00 & 12.80 & 4.00 & 2.60 \\
\hline 01-08-2017 & 31 & 18.56 & 2.17 & 29.90 & 19.70 & 85.00 & 50.00 & 10.40 & 8.10 & 2.40 \\
\hline 08-08-2017 & 32 & 16.61 & 2.21 & 27.10 & 19.30 & 93.00 & 57.00 & 4.80 & 2.60 & 4.60 \\
\hline 15-08-2017 & 33 & 15.35 & 3.13 & 29.00 & 20.50 & 89.00 & 55.00 & 6.90 & 4.10 & 70.40 \\
\hline 22-08-2017 & 34 & 13.34 & 3.31 & 27.50 & 19.70 & 92.00 & 64.00 & 6.30 & 3.10 & 34.20 \\
\hline 29-08-2017 & 35 & 13.01 & 3.41 & 27.50 & 19.70 & 90.00 & 59.00 & 8.20 & 3.60 & 62.80 \\
\hline 05-09-2017 & 36 & 16.23 & 3.49 & 27.20 & 19.80 & 95.00 & 58.00 & 9.40 & 3.00 & 30.80 \\
\hline 12-09-2017 & 37 & 17.11 & 3.52 & 28.30 & 19.50 & 89.00 & 56.00 & 4.20 & 5.30 & 99.20 \\
\hline 19-09-2017 & 38 & 17.27 & 3.17 & 28.80 & 19.30 & 89.00 & 57.00 & 6.40 & 5.20 & 24.20 \\
\hline 26-09-2017 & 39 & 18.12 & 3.15 & 28.10 & 18.80 & 92.00 & 65.00 & 9.70 & 3.60 & 24.40 \\
\hline
\end{tabular}


Table.6a Incidence of blotch miner and spiralling whitefly caused by Acrocercops anthrauris Fabricius in relation to weather parameters of the corresponding week during 2016 -17 (Nursery)

\begin{tabular}{|c|c|c|c|c|c|c|c|c|c|c|}
\hline \multirow{2}{*}{$\begin{array}{c}\text { Date of } \\
\text { observation }\end{array}$} & \multirow{2}{*}{$\begin{array}{l}\text { Std. } \\
\text { Met. } \\
\text { week }\end{array}$} & \multirow{2}{*}{$\begin{array}{c}\text { No. of } \\
\text { blotches/ } \\
\text { leaf }\end{array}$} & \multirow{2}{*}{$\begin{array}{c}\text { No. of egg } \\
\text { spirals of } \\
\text { SWF/leaf }\end{array}$} & \multicolumn{2}{|c|}{ Temp $\left({ }^{0} \mathrm{C}\right)$} & \multicolumn{2}{|c|}{ RH (\%) } & \multirow{2}{*}{$\begin{array}{c}\text { Wind } \\
\text { speed } \\
(\mathrm{Km} / \text { day })\end{array}$} & \multirow{2}{*}{$\begin{array}{c}\text { Bright } \\
\text { Sunshine } \\
\text { (Hrs) }\end{array}$} & \multirow{2}{*}{$\begin{array}{c}\text { Total } \\
\text { Rainfall } \\
(\mathrm{mm})\end{array}$} \\
\hline & & & & Max. & Min. & Morning & $\mathbf{A} / \mathbf{N}$ & & & \\
\hline 3-10-2016 & 40 & 17.72 & 3.13 & 29.91 & 18.91 & 92.00 & 47.29 & 5.60 & 8.30 & 0.00 \\
\hline $10-10-2016$ & 41 & 17.83 & 3.63 & 29.09 & 18.66 & 92.00 & 50.57 & 5.50 & 4.90 & 30.20 \\
\hline $17-10-2016$ & 42 & 18.03 & 3.01 & 29.74 & 16.69 & 77.86 & 45.57 & 3.40 & 9.20 & 0.00 \\
\hline 24-10-2016 & 43 & 18.39 & 3.43 & 29.66 & 17.4 & 76.71 & 45.86 & 3.70 & 8.80 & 0.00 \\
\hline $31-10-2016$ & 44 & 17.49 & 2.63 & 29.69 & 18.11 & 85.00 & 47.14 & 4.00 & 8.00 & 0.00 \\
\hline 7-11-2016 & 45 & 16.64 & 2.61 & 29.20 & 15.97 & 78.71 & 45.00 & 4.70 & 9.60 & 0.00 \\
\hline $14-11-2016$ & 46 & 15.83 & 2.53 & 29.46 & 18.43 & 84.14 & 45.71 & 7.70 & 7.20 & 0.00 \\
\hline 21-11-2016 & 47 & 14.99 & 2.47 & 29.89 & 15.37 & 82.00 & 38.43 & 6.70 & 8.70 & 0.00 \\
\hline 28-11-2016 & 48 & 13.53 & 2.41 & 29.49 & 14.00 & 82.57 & 82.57 & 4.00 & 8.70 & 1.70 \\
\hline $5-12-2016$ & 49 & 10.24 & 2.39 & 27.37 & 15.6 & 82.57 & 43.14 & 7.70 & 4.70 & 0.00 \\
\hline 12-12-2016 & 50 & 7.93 & 2.32 & 24.80 & 15.11 & 89.86 & 56.00 & 7.20 & 4.70 & 58.20 \\
\hline $19-12-2016$ & 51 & 2.95 & 2.23 & 28.14 & 13.91 & 87.43 & 41.14 & 5.50 & 8.60 & 0.00 \\
\hline $26-12-2016$ & 52 & 1.99 & 2.17 & 27.91 & 13.60 & 90.71 & 39.29 & 5.10 & 9.70 & 0.00 \\
\hline 2-01-2017 & 1 & 1.01 & 1.91 & 27.37 & 12.17 & 85.86 & 41.00 & 5.20 & 9.40 & 0.00 \\
\hline 9-01-2017 & 2 & 0.00 & 1.63 & 27.23 & 15.11 & 91.14 & 41.86 & 5.10 & 8.20 & 0.00 \\
\hline 16-01-2017 & 3 & 0.00 & 0.00 & 26.77 & 14.09 & 93.14 & 39.43 & 8.50 & 9.40 & 0.00 \\
\hline 23-01-2017 & 4 & 0.00 & 0.00 & 27.29 & 15.43 & 85.57 & 38.57 & 9.70 & 5.80 & 0.00 \\
\hline 30-01-2017 & 5 & 0.00 & 0.00 & 27.29 & 15.43 & 85.57 & 38.57 & 4.80 & 10.50 & 0.00 \\
\hline 6-02-2017 & 6 & 0.00 & 0.00 & 29.89 & 13.89 & 82.86 & 34.00 & 6.50 & 10.30 & 0.00 \\
\hline 13-02-2017 & 7 & 0.21 & 0.00 & 29.43 & 14.63 & 83.86 & 33.86 & 10.20 & 10.00 & 0.00 \\
\hline 20-02-2017 & 8 & 3.94 & 0.00 & 31.94 & 15.77 & 84.71 & 35.71 & 5.90 & 9.90 & 1.40 \\
\hline 27-02-2017 & 9 & 7.82 & 0.00 & 31.97 & 15.03 & 78.71 & 29.14 & 5.90 & 9.00 & 0.00 \\
\hline 6-03-2017 & 10 & 13.33 & 1.05 & 32.29 & 20.06 & 86.57 & 41.29 & 6.20 & 8.40 & 1.40 \\
\hline \multirow{2}{*}{$\begin{array}{c}\text { Date of } \\
\text { observation }\end{array}$} & \multirow{2}{*}{$\begin{array}{l}\text { Std. } \\
\text { Met. } \\
\text { week }\end{array}$} & \multirow{2}{*}{$\begin{array}{c}\text { No. of } \\
\text { blotches/ } \\
\text { leaf }\end{array}$} & \multirow{2}{*}{$\begin{array}{l}\text { No. of egg } \\
\text { spirals of } \\
\text { SWF/leaf }\end{array}$} & \multicolumn{2}{|c|}{ Temp ${ }^{0} \mathrm{C}$} & \multicolumn{2}{|c|}{ RH\% } & Wind & Bright & Total \\
\hline & & & & Max. & Min. & Morning & $\mathbf{A} / \mathbf{N}$ & $\begin{array}{c}\text { speed } \\
\text { (Km/day) }\end{array}$ & $\begin{array}{l}\text { Sunshin } \\
\text { e (Hrs) }\end{array}$ & $\begin{array}{l}\text { Raintal } \\
\text { l(mm) }\end{array}$ \\
\hline
\end{tabular}




\begin{tabular}{|c|c|c|c|c|c|c|c|c|c|c|}
\hline 13-03-2017 & 11 & 14.56 & 1.13 & 32.26 & 19.94 & 83.57 & 41.57 & 6.50 & 8.50 & 0.00 \\
\hline 20-03-2017 & 12 & 15.34 & 1.21 & 33.40 & 20.49 & 78.43 & 40.43 & 6.90 & 9.40 & 0.00 \\
\hline 27-03-2017 & 13 & 16.86 & 2.32 & 35.00 & 20.50 & 82.00 & 35.00 & 7.70 & 9.30 & 0.00 \\
\hline 2-04-2017 & 14 & 17.97 & 2.66 & 34.00 & 21.50 & 82.00 & 38.00 & 6.20 & 8.60 & 1.60 \\
\hline 9-04-2017 & 15 & 18.13 & 2.55 & 35.70 & 22.40 & 82.00 & 36.00 & 6.70 & 8.50 & 0.00 \\
\hline 16-04-2017 & 16 & 18.97 & 2.43 & 34.60 & 21.90 & 84.00 & 36.00 & 8.40 & 7.70 & 0.00 \\
\hline 23-04-2017 & 17 & 20.14 & 2.51 & 35.40 & 22.40 & 84.00 & 36.00 & 7.00 & 8.80 & 0.00 \\
\hline 30-04-2017 & 18 & 23.24 & 2.63 & 32.70 & 20.90 & 82.00 & 31.00 & 6.00 & 8.20 & 25.80 \\
\hline 7-05-2017 & 19 & 25.36 & 2.91 & 32.60 & 21.20 & 81.00 & 48.00 & 6.40 & 6.90 & 15.20 \\
\hline 14-05-2017 & 20 & 26.53 & 3.01 & 33.50 & 20.90 & 85.00 & 35.00 & 8.30 & 8.00 & 45.60 \\
\hline 21-05-2017 & 21 & 29.88 & 3.12 & 32.20 & 19.80 & 87.00 & 42.00 & 8.00 & 6.50 & 133.60 \\
\hline 28-05-2017 & 22 & 31.33 & 4.11 & 30.00 & 19.90 & 88.00 & 47.00 & 10.80 & 8.20 & 8.30 \\
\hline 4-06-2017 & 23 & 32.09 & 4.68 & 29.50 & 20.60 & 88.00 & 53.00 & 10.20 & 6.80 & 21.60 \\
\hline 11-06-2017 & 24 & 30.21 & 4.31 & 28.10 & 20.20 & 90.00 & 57.00 & 10.90 & 2.30 & 8.00 \\
\hline 18-06-2017 & 25 & 28.24 & 4.16 & 28.10 & 19.50 & 90.00 & 56.00 & 9.00 & 4.80 & 32.60 \\
\hline 25-06-2017 & 26 & 26.13 & 3.32 & 28.30 & 20.00 & 87.00 & 54.00 & 13.30 & 5.10 & 3.20 \\
\hline 2-07-2017 & 27 & 25.42 & 3.41 & 29.00 & 19.70 & 93.00 & 57.00 & 9.60 & 4.20 & 10.20 \\
\hline 9-07-2017 & 28 & 24.01 & 3.29 & 28.00 & 19.80 & 90.00 & 61.00 & 9.80 & 3.70 & 13.40 \\
\hline 16-07-2017 & 29 & 22.34 & 2.11 & 27.50 & 19.60 & 88.00 & 59.00 & 12.80 & 4.00 & 2.60 \\
\hline 23-07-2017 & 30 & 20.77 & 2.04 & 29.90 & 19.70 & 85.00 & 50.00 & 10.40 & 8.10 & 2.40 \\
\hline 01-08-2017 & 31 & 18.56 & 2.17 & 27.10 & 19.30 & 93.00 & 57.00 & 4.80 & 2.60 & 4.60 \\
\hline 08-08-2017 & 32 & 16.61 & 2.21 & 29.00 & 20.50 & 89.00 & 55.00 & 6.90 & 4.10 & 70.40 \\
\hline 15-08-2017 & 33 & 15.35 & 3.13 & 27.50 & 19.70 & 92.00 & 64.00 & 6.30 & 3.10 & 34.20 \\
\hline 22-08-2017 & 34 & 13.34 & 3.31 & 27.50 & 19.70 & 90.00 & 59.00 & 8.20 & 3.60 & 62.80 \\
\hline 29-08-2017 & 35 & 13.01 & 3.41 & 27.20 & 19.80 & 95.00 & 58.00 & 9.40 & 3.00 & 30.80 \\
\hline 05-09-2017 & 36 & 16.23 & 3.49 & 28.30 & 19.50 & 89.00 & 56.00 & 4.20 & 5.30 & 99.20 \\
\hline 12-09-2017 & 37 & 17.11 & 3.52 & 28.80 & 19.30 & 89.00 & 57.00 & 6.40 & 5.20 & 24.20 \\
\hline 19-09-2017 & 38 & 17.27 & 3.17 & 28.10 & 18.80 & 92.00 & 65.00 & 9.70 & 3.60 & 24.40 \\
\hline 26-09-2017 & 39 & 18.12 & 3.15 & 27.30 & 19.10 & 90.00 & 60.00 & 4.30 & 4.00 & 108.60 \\
\hline
\end{tabular}


Table.7 Correlation matrix between incidence of blotch miner and spiralling whitefly weather parameters of preceding week (Nursery)

\begin{tabular}{|c|c|c|c|c|c|c|c|}
\hline \multirow{2}{*}{ Pest/Damage } & \multicolumn{2}{|c|}{ Temp $\left({ }^{0} \mathrm{C}\right)$} & \multicolumn{2}{|c|}{ RH (\%) } & \multirow{2}{*}{$\begin{array}{c}\text { Wind speed } \\
(\mathrm{Km} / \text { day })\end{array}$} & \multirow{2}{*}{$\begin{array}{c}\text { Bright } \\
\text { Sunshine } \\
\text { (Hrs) }\end{array}$} & \multirow{2}{*}{$\begin{array}{c}\text { Total } \\
\text { Rainfall } \\
(\mathrm{mm})\end{array}$} \\
\hline & Max. & Min. & Morning & $\mathbf{A} / \mathbf{N}$ & & & \\
\hline Blotches/ leaf & $0.413 * *$ & $0.684 * *$ & -0.022 & $0.290 *$ & $0.361 * *$ & $-0.324 *$ & $0.292 *$ \\
\hline Egg spirals/leaf & $0.072 * *$ & $0.517 * *$ & 0.181 & $0.520 * *$ & 0.181 & $-0.484 * *$ & 0.437 \\
\hline
\end{tabular}

Table.8 Correlation matrix between incidence of blotch miner and spiralling whitefly with weather parameters of corresponding week (Nursery)

\begin{tabular}{|c|c|c|c|c|c|c|c|}
\hline \multirow{2}{*}{ Pest/Damage } & \multicolumn{2}{|c|}{ Temp $\left({ }^{0} \mathrm{C}\right)$} & \multicolumn{2}{|c|}{$\mathrm{RH}(\%)$} & \multirow{2}{*}{$\begin{array}{c}\text { Wind } \\
\text { speed } \\
(\mathrm{Km} / \text { day })\end{array}$} & \multirow{2}{*}{$\begin{array}{l}\text { Bright } \\
\text { Sunshine } \\
\text { (Hrs) }\end{array}$} & \multirow{2}{*}{$\begin{array}{c}\text { Total } \\
\text { Rainfall } \\
(\mathrm{mm})\end{array}$} \\
\hline & Max. & Min. & Morning & $\mathbf{A} / \mathbf{N}$ & & & \\
\hline Blotches/ leaf & $0.289 *$ & $0.775^{* *}$ & 0.030 & $0.336^{*}$ & $0.359 * *$ & $-0.409 * *$ & $0.281 *$ \\
\hline Egg spirals/leaf & $0.069 * *$ & $0.523 * *$ & $0.277 *$ & $0.536^{* *}$ & 0.157 & -0.537 & $0.353 *$ \\
\hline
\end{tabular}

Table.9 Multiple linear regression between incidence of blotch miner and spiralling whitefly with weather parameters of preceding week (Nursery)

\begin{tabular}{|c|l|l|}
\hline Pest & \multicolumn{1}{|c|}{ Regression equation } & \multicolumn{1}{|c|}{$\mathrm{R}^{2}$} \\
\hline Blotches/ leaf & $\begin{array}{l}\mathrm{Y}=-68.001+2.178 \mathrm{R}_{1}+0.611 \mathrm{R}_{2}-0.293 \mathrm{R}_{3}+ \\
0.561 \mathrm{R}_{4}+0.893 \mathrm{R}_{5}-0.038 \mathrm{R}_{6}+0.052 \mathrm{R}_{7}\end{array}$ & 70.10 \\
\hline Spirals/leaf & $\begin{array}{l}\mathrm{Y}=-5.372+0.157 \mathrm{R}_{1}+0.083 \mathrm{R}_{2}-0.031 \mathrm{R}_{3}+ \\
0.089 \mathrm{R}_{4}-0.009 \mathrm{R}_{5}+0.005 \mathrm{R}_{6}+0.011 \mathrm{R}_{7}\end{array}$ & 56.40 \\
\hline
\end{tabular}

Table.10 Multiple linear regression between incidence of blotch miner and spiralling whitefly weather parameters of corresponding week (Nursery)

\begin{tabular}{|c|c|c|}
\hline Pest & Regression equation & \multicolumn{2}{|c|}{$\mathrm{R}^{2}$} \\
\hline Blotches/ leaf & $\mathrm{Y}=-0.498-0.236 \mathrm{R}_{1}+2.457 \mathrm{R}_{2}-0.509 \mathrm{R}_{3}+$ & 71.50 \\
& $\left.0.284 \mathrm{R}_{4}+0.767 \mathrm{R}_{5}+0.385 \mathrm{R}_{6}+0.037 \mathrm{R}_{7}\right)$ & \\
\hline Spirals/leaf & $\mathrm{Y}=0.339-0.114 \mathrm{R}_{1}+0.280 \mathrm{R}_{2}-0.018 \mathrm{R}_{3}+0.042 \mathrm{R}_{4}$ & 51.80 \\
& $\left.-0.035 \mathrm{R}_{5}+0.019 \mathrm{R}_{6}+0.003 \mathrm{R}_{7}\right)$ & \\
\hline
\end{tabular}


Incidence of blotch miner and spiralling whitefly in relation to weather parameters in Nursery

Maximum blotch miner population was observed in I week of June (32.09), followed by last week of May (31.33).

Maximum spiralling whitefly population (i.e. egg spiral number) was observed in I week of June (4.68), followed by II week of June (4.31) (Table 6 and 6a).

Correlation between incidence of Blotch miner, Spiralling whitefly and Flower galls with weather parameters of preceding week in Nursery

The correlation analysis between number of leaf blotches with weather parameters of preceding week (Table 7) was found to be highly significant and positively correlated with maximum temperature $(\mathrm{r}=0.413$, $\mathrm{p}<0.01)$, minimum temperature $(\mathrm{r}=0.684$, $\mathrm{p}<0.01)$, wind speed $(\mathrm{r}=0.361 * *, \mathrm{p}<0.01)$, afternoon RH $(r=0.290, p<0.05)$ and total rainfall $(r=0.292, p<0.05)$.

Negative correlation existed between blotch number and sunshine hrs. ( $\mathrm{r}=-0.324)$ and morning RH $(r=-0.022)$, but this relationship was non - significant.

The correlation between spiralling whitefly with weather parameters of preceding week (Table 7) was found to be highly significant and positively correlated with maximum temperature $(r=0.072, p<0.01)$, minimum temperature i.e. $(\mathrm{r}=0.517, \mathrm{p}<0.01)$ and afternoon $\mathrm{RH}(\mathrm{r}=0.520, \mathrm{p}<0.01)$.

Non significant, positive correlation existed with rainfall $(\mathrm{r}=0.437)$, morning $\mathrm{RH}(\mathrm{r}=$ $0.181)$ and wind speed $(r=0.181)$ but non significant negative correlation was observed with sunshine hrs $(r=-0.484)$.
Correlation matrix between incidence of blotch miner and spiralling whitefly with weather parameters of corresponding week in Nursery

The correlation between leaf blotches with weather parameters of corresponding week (Table 8) was found to be significant and positive with minimum temperature $(\mathrm{r}=0.775$, $\mathrm{p}<0.01)$, maximum temperature $(\mathrm{r}=0.289$, $\mathrm{p}<0.05)$, afternoon relative humidity ( $\mathrm{r}=$ $0.336, \mathrm{p}<0.05)$ and wind speed $(r=0.359$, $\mathrm{p}<0.01)$. However it was significantly negatively correlated with sunshine $\mathrm{hrs}(\mathrm{r}=--$ $0.409, \mathrm{p}<0.01)$ and non - significant with morning relative humidity $(\mathrm{r}=0.030)$.

The correlation between spiralling whitefly with weather parameters of preceeding week (Table 7) was found to be highly significant and positively correlated with maximum temperature $(\mathrm{r}=0.069, \mathrm{p}<0.01)$, minimum temperature i.e. $\left(\mathrm{r}=0.523^{* *}, \mathrm{p}<0.01\right)$ morning $\mathrm{RH}(\mathrm{r}=0.277, \mathrm{p}<0.05)$ afternoon $\mathrm{RH}(\mathrm{r}=$ $\left.0.536^{* *}, \mathrm{p}<0.01\right)$ and rainfall $(\mathrm{r}=0.353$, $\mathrm{p}<0.05)$, Non significant, positive correlation existed with and wind speed $(r=0.181)$ but non - significant negative correlation was observed with sunshine hrs $(\mathrm{r}=-0.537){ }^{[2]}$ substantiating that intensive study on the incidence of whitefly family Aleyrodidae was Undertaken at monthly intervals and the weather condition was recorded. The incidence of whitefly was maximum in May and June months, it is due to suitable temperatures for whitefly population.

The mean maximum population of blotch miner was observed in the provenance Jabalpur which was recorded 8.41 blotches/leaf and minimum in the provenance Zaheerabad which was recorded as 3.33 blotch/leaf during August to April. Hence, it can be concluded that Jabalpur provenance was more susceptible and Zaheerabad provenance was less susceptible to the attack of leaf blotch miner ${ }^{[6]}$. 
Multiple linear regression between incidence of blotch miner and spiralling whitefly with weather parameters of preceding week in Nursery

Multiple linear regression revealed that the weather parameters of preceding week influenced blotch miner and spiralling whitefly incidence to an extent of 70.10 and 56.40 per cent, respectively.

Multiple linear regression between incidence of blotch miner and spiralling whitefly with weather parameters of corresponding week in Nursery

Multiple linear regression revealed that the weather parameters of corresponding week influenced blotch miner and spiralling whitefly incidence to an extent of 71.50 and 51.80 per cent, respectively (Table 9 and 10).

According to ${ }^{[1]}$ the initiation of Aceria pongamiae infestation was recorded during February, reaching peak population levels by May. The decline in mite population during June would be reflecting the negative impact of rainfall received by the site. Even though the mites enjoy a highly secluded habitat available within the leaf galls, rainfall exerts an adverse effect by penetrating through the holes into the interior of the gall cavity. But the results of the present study are not in conformity with findings of ${ }^{[1]}$ which could be due to differences in geographical location and variations in the environmental conditions and crop situations.

\section{Acknowledgement}

The authors wish to express their heartfelt gratitude to the Indian Council of Agricultural Research, New Delhi, for providing financial support and Dr. N. Srinivasa and Dr. C. Chinnamade Gowda Professors, AINP (Agricultural Acarology), UAS, GKVK,
Bengaluru for providing facilities for conducting this investigation and in taking scanning electron micrographs of the mite

\section{References}

1. Aratchige NS, Fernando LCP. Waidyarathne, KP. Population dynamics of Aceria guerreronis (Acari: Eriophyidae) and its predatory mite, Neoseiulus baraki (Acari: Phytoseiidae) in two coconut growing areas in Sri Lanka. Exp Appl Acarol., 2012; 56:319-325.

2. Chikkaswamy BK, Rabin CP. Studies on the incidence of white fly in Pongamia tree garden. Int. J. Curr. Microbiol. App. Sci., 2014; 3 (6): 1001-1003

3. Naik M, Meher, LC, Naik SN. Dasa LM. Production of biodiesel from high free fatty acid Karanja (Pongamia pinnata) oil. Biomass and Bio energy., 2008; 32: 354-7

4. Nasareen PNM, Ramani N. Seasonal variation in the population density of the gall mite, Aceria pongamiae Keifer (Acari: Eriophyidae) within the leaf galls of Pongamia pinnata (L.). JEZS., 2014; 2 (3): 126-130

5. Sahadev C, Yousuf M, Gupta RK. Loss assessment in Pongamia pinnata, caused by Aceria pongamiae (Acarina: Eriophyidae). Journal Vaniki Sandesh., 2009; 33: 9-12.

6. Sahu C, Ganguli JL, Kerketta A, Painkra KL. Screening of Karanj, Pongamia pinnata L. against various insect pests under agro - silvi pattern of cropping system at Raipur (C.G.). Int. J. of plant prot., 2016; 9 (2): 570-577.

7. Sangwan S, Rao DV, Sharma RA. A review on Pongamia pinnata (L.) Pierre: A great versatile leguminous plant, Nat. \& Sci., 2010; 8 (11): 130139.

8. Sundararaj R, Rajamuthukrishnan, 
Ramadevi OK. Annotated list of insect in India. Annals of Forestry., 2005; pests of Pongamia pinnata (L.) Pierre 13: 337-341.

\section{How to cite this article:}

Devika Rani, D., D. Jemla Naik and Jagadish, K.S. 2018. Seasonal Incidence of Major Pests of Pongamia (Milletia pinnata L.) in Nursery Conditions. Int.J.Curr.Microbiol.App.Sci. 7(08): 2337-2350. doi: https://doi.org/10.20546/ijcmas.2018.708.235 